\title{
EXTENSIONS OF SEVERAL CLASSICAL RESULTS FOR INDEPENDENT AND IDENTICALLY DISTRIBUTED RANDOM VARIABLES TO CONDITIONAL CASES
}

\author{
De-Mei Yuan and Shun-Jing Li
}

\begin{abstract}
Extensions of the Kolmogorov convergence criterion and the Marcinkiewicz-Zygmund inequalities from independent random variables to conditional independent ones are derived. As their applications, a conditional version of the Marcinkiewicz-Zygmund strong law of large numbers and a result on convergence in $L^{p}$ for conditionally independent and conditionally identically distributed random variables are established, respectively.
\end{abstract}

\section{Introduction}

We will be working on a fixed probability space $(\Omega, \mathcal{A}, P)$ and let $\mathcal{F}$ be a sub- $\sigma$-algebra of $\mathcal{A}$. A finite sequence $\left\{X_{k}, 1 \leq k \leq n\right\}$ of random variables is said to be conditionally independent given $\mathcal{F}(\mathcal{F}$-independent, in short) if

$$
P\left\{\bigcap_{k=1}^{n}\left(X_{k} \in B_{k}\right) \mid \mathcal{F}\right\}=\prod_{k=1}^{n} P\left(X_{k} \in B_{k} \mid \mathcal{F}\right) \text { a.s. }
$$

for any choice of finitely many sets $B_{1}, B_{2}, \ldots, B_{n} \in \mathcal{B}$, where $\mathcal{B}$ is the Borel $\sigma$-algebra in $\mathbb{R}$. An infinite sequence $\left\{X_{n}, n \geq 1\right\}$ of random variables is said to be $\mathcal{F}$-independent if every finite subsequence is $\mathcal{F}$-independent.

Of course, $\mathcal{F}$-independence reduces to the usual (unconditional) independence if $\mathcal{F}=\{\varnothing, \Omega\}$ is the trivial $\sigma$-algebra. In Prakasa Rao [9], concrete

Received July 15, 2014; Revised October 26, 2014.

2010 Mathematics Subject Classification. 60F15, 60F25.

Key words and phrases. conditional independence, conditional identical distribution, conditional Kolmogorov convergence criterion, conditional Marcinkiewicz-Zygmund inequalities, conditional Marcinkiewicz-Zygmund strong law.

The authors would like to thank the anonymous referees sincerely for their valuable comments and a well-meaning reminder of a missing reference. This work was supported by National Natural Science Foundation of China (No.11101452), SCR of Chongqing Municipal Education Commission (No. KJ1400613) and Natural Science Foundation Project of CTBU of China (No.1352001). 
examples were given, where independent random variables lose their independence under conditioning, and dependent random variables become independent under conditioning.

We next recall the concept of conditionally identical distribution. Two random variables $X$ and $Y$ are said to be conditionally identically distributed given $\mathcal{F}(\mathcal{F}$-identically distributed, in short $)$ if

$$
P(X \in B \mid \mathcal{F})=P(Y \in B \mid \mathcal{F}) \text { a.s. for any } B \in \mathcal{B} \text {. }
$$

A collection of random variables is said to be $\mathcal{F}$-identically distributed if every pair of random variables in the collection is $\mathcal{F}$-identically distributed.

Conditionally identical distribution, just like conditional independence, reduces to the usual identical distribution if the conditional $\sigma$-algebra $\mathcal{F}=$ $\{\varnothing, \Omega\}$. It is easily shown that conditionally identical distribution implies identical distribution, but the converse implication need not always be true, such counterexamples can be found in Yuan et al. [18] and Roussas [12].

Let $\left\{X_{n}, n \geq 1\right\}$ be exchangeable random variables, that is, the joint distribution of $\left(X_{1}, X_{2}, \ldots, X_{n}\right)$ is the same as that of $\left(X_{\pi(1)}, X_{\pi(2)}, \ldots, X_{\pi(n)}\right)$ for each $n \geq 1$ and any permutation $\pi$ of $\{1,2, \ldots, n\}$. By de Finetti's theorem, $\left\{X_{n}\right\}$ is conditionally independent and conditionally identically distributed given either its tail $\sigma$-algebra or the $\sigma$-algebra of permutable events, c.f. Theorem 7.3.2 of Chow and Teicher [2].

The statistical perspective of conditional independence and conditionally identical distribution is that of a Bayesian. A problem begins with a parameter $\theta$ with its prior probability distribution that exists only in mind of the investigator. The statistical model that is most commonly in use is that of a sequence $\left\{X_{n}, n \geq 1\right\}$ of observable random variables that is independent and identically distributed for each given value of $\theta$. As such, $\left\{X_{n}, n \geq 1\right\}$ is $\mathcal{F}$ independent and $\mathcal{F}$ - identically distributed but neither necessarily independent nor necessarily identically distributed, where $\mathcal{F}=\sigma(\theta)$.

Let $\left\{X_{n}, n \geq 1\right\}$ be a sequence of random variables and $S_{n}=\sum_{k=1}^{n} X_{k}$. Majerek et al. [7] proved that if $\left\{X_{n}, n \geq 1\right\}$ is $\mathcal{F}$-independent and $\mathcal{F}$-identically distributed, then

$$
\lim _{n \rightarrow \infty} n^{-1} S_{n}=Y \text { a.s. }
$$

if and only if $E^{\mathcal{F}} X=Y$ a.s., which is a conditional version of the Kolmogorov strong law of large numbers.

The further derivations are conditional versions of the generalized Kolmogorov inequality and Hájek-Rényi inequality due to Prakasa Rao [9] as well as conditional central limit theorems due to Yuan et al. [18].

Hence we are just wondering which results on independent and identically distributed random variables have analogous ones in a conditional setting? As pointed out by Prakasa Rao [9], one does have to derive results under conditioning if there is a need even though the results and proofs of such results may be analogous to those under the non-conditioning setup. This motivates our 
original interest in investigating conditionally independent and conditionally identically distributed random variables.

Starting from the conditional independence given a sub- $\sigma$-algebra $\mathcal{F}$, the past nearly a decade has witnessed the active development of a rich probability theory of conditional dependence and many important theoretical results have been obtained. See, for instance, Christofides and Hadjikyriakou [3] for conditional demimartingale, Liu and Prakasa Rao [5] for conditional Borel-Cantelli lemma, Ordóñez Cabrera et al. [8] for conditionally negatively quadrant dependence, Wang and Wang [14] for conditional demimartingale and conditional N-demimartingale, Yuan et al. [15] for conditionally negative association, Yuan and Lei [17] for conditionally strong mixing, Yuan et al. [16] for conditionally uniformly strong mixing, Yuan and Xie [19] for conditionally linearly negatively quadrant dependence, Yuan and Yang [20] for conditional association. These achievements also stimulate us to study conditionally independent and conditionally identically distributed random variables.

In Section 2, we first derive an extension of the Kolmogorov convergence criterion for independent random variables to conditional case, and then, as its application, establish a conditional version of the Marcinkiewicz-Zygmund strong law of large numbers for conditionally independent and conditionally identically distributed random variables. An extension of the MarcinkiewiczZygmund inequalities and a result on convergence in $L^{p}$ as its application are studied in Section 3.

Following Prakasa Rao [9], for the sake of convenience, we will use the notation $P^{\mathcal{F}}(A)$ to denote $P(A \mid \mathcal{F})$ and $E^{\mathcal{F}} X$ to denote $E(X \mid \mathcal{F})$. In addition, $\operatorname{Var}^{\mathcal{F}} X$ stands for the conditional variance of $X$ given $\mathcal{F}$, that is,

$$
\operatorname{Var}^{\mathcal{F}} X=E^{\mathcal{F}}\left(X-E^{\mathcal{F}} X\right)^{2} .
$$

\section{Conditional versions of Kolmogorov convergence criterion and Marcinkiewicz-Zygmund strong law}

Our first result is not only a conditional version of the Kolmogorov convergence criterion, but also an extension of Theorem 3.5 appeared in Majerek et al. [7]. The authors did not know this criterion has appeared in Liu and Zhang [6] until revising the paper, but the proof here follows a different route from that paper.

Theorem 2.1. If $\left\{X_{n}, n \geq 1\right\}$ is a sequence of $\mathcal{F}$-independent random variables such that $\sum_{n=1}^{\infty} \operatorname{Var}^{\mathcal{F}} X_{n}<\infty$ a.s., then $S_{n}-E^{\mathcal{F}} S_{n}$ converges almost surely.

Proof. For any positive integers $m$ and $n$ with $m>n$, applying the conditional version of Kolomogorov's inequality, Theorem 3.4 in Majerek et al. [7], to the 
sequence $X_{n+1}, \ldots, X_{m}$, we find

$$
\begin{aligned}
& P^{\mathcal{F}}\left(\max _{n+1 \leq k \leq m}\left|\left(S_{k}-E^{\mathcal{F}} S_{k}\right)-\left(S_{n}-E^{\mathcal{F}} S_{n}\right)\right|>\varepsilon\right) \\
= & P^{\mathcal{F}}\left(\max _{n+1 \leq k \leq m}\left|\sum_{j=n+1}^{k}\left(X_{j}-E^{\mathcal{F}} X_{j}\right)\right|>\varepsilon\right) \\
\leq & \varepsilon^{-2} \sum_{k=n+1}^{m} \operatorname{Var}^{\mathcal{F}} X_{k}
\end{aligned}
$$

for any $\varepsilon>0$. Letting $m$ approach infinity, we have

$$
P^{\mathcal{F}}\left(\sup _{k \geq n+1}\left|\left(S_{k}-E^{\mathcal{F}} S_{k}\right)-\left(S_{n}-E^{\mathcal{F}} S_{n}\right)\right|>\varepsilon\right) \leq \varepsilon^{-2} \sum_{k=n+1}^{\infty} \operatorname{Var}^{\mathcal{F}} X_{k} .
$$

In view of the assumption that $\sum_{n=1}^{\infty} \operatorname{Var}^{\mathcal{F}} X_{n}<\infty$ a.s., this implies that

$$
P^{\mathcal{F}}\left(\sup _{k \geq n+1}\left|\left(S_{k}-E^{\mathcal{F}} S_{k}\right)-\left(S_{n}-E^{\mathcal{F}} S_{n}\right)\right|>\varepsilon\right) \rightarrow 0 \text { a.s. as } n \rightarrow \infty,
$$

which together with the dominated convergence theorem means

$$
P\left(\sup _{k \geq n+1}\left|\left(S_{k}-E^{\mathcal{F}} S_{k}\right)-\left(S_{n}-E^{\mathcal{F}} S_{n}\right)\right|>\varepsilon\right) \rightarrow 0 \text { as } n \rightarrow \infty .
$$

By Theorem 2.10.1 of Shiryaev [13], the sequence $\left\{S_{n}-E^{\mathcal{F}} S_{n}, n \geq 1\right\}$ is fundamental with probability 1 , so that $S_{n}-E^{\mathcal{F}} S_{n}$ converges almost surely.

As usual, $I_{A}$ denotes the indicator function of an event $A$ and sometimes it is written as $I(A)$. As an application of Theorem 2.1, a conditional version of the Marcinkiewicz and Zygmund strong law of large numbers can be established.

Theorem 2.2. Let $0<p<2$. Suppose that $\left\{X_{n}, n \geq 1\right\}$ is a sequence of $\mathcal{F}$ independent and $\mathcal{F}$-identically distributed random variables with $E^{\mathcal{F}}\left|X_{1}\right|^{p}<\infty$ a.s. and $E^{\mathcal{F}} X_{1}=0$ a.s. when $1 \leq p<2$. Then

$$
n^{-1 / p} S_{n} \rightarrow 0 \text { a.s. }
$$

In order to prove the above theorem, let us first give two lemmas. It is worthy to note that Lemma 2.4 below serves not only the proof of Theorem 2.2 , but also that of Theorem 2.6.

Lemma 2.3. Let $0<p<2$. Suppose that $\left\{X_{n}, n \geq 1\right\}$ is a sequence of $\mathcal{F}$ independent and $\mathcal{F}$-identically distributed random variables, and set

$$
Y_{n}=X_{n} I\left(\left|X_{n}\right| \leq n^{1 / p}\right), n \geq 1 .
$$

If $E^{\mathcal{F}}\left|X_{1}\right|^{p}<\infty$ a.s., then

$$
\sum_{n=1}^{\infty} \operatorname{Var}^{\mathcal{F}}\left(n^{-1 / p} Y_{n}\right)<\infty \text { a.s. }
$$


Proof. If $\alpha>0$ and $n \geq 1$, then

$$
\frac{1}{\alpha n^{\alpha}}=\int_{n}^{\infty} \frac{d x}{x^{\alpha+1}}=\sum_{k=n}^{\infty} \int_{k}^{k+1} \frac{d x}{x^{\alpha+1}} \geq \sum_{k=n}^{\infty} \frac{1}{(k+1)^{\alpha+1}}=\sum_{k=n+1}^{\infty} \frac{1}{k^{\alpha+1}} .
$$

Suppose, further, that $n \geq 2$, then

$$
\sum_{k=n}^{\infty} \frac{1}{k^{\alpha+1}} \leq \frac{1}{\alpha(n-1)^{\alpha}} \leq \frac{2^{\alpha}}{\alpha n^{\alpha}}
$$

Exploiting this relation and employing the slicing technique, we have

$$
\begin{aligned}
& \sum_{n=1}^{\infty} \operatorname{Var}^{\mathcal{F}}\left(n^{-1 / p} Y_{n}\right) \\
& \leq \sum_{n=1}^{\infty} n^{-2 / p} E^{\mathcal{F}} Y_{n}^{2} \\
& =\sum_{n=1}^{\infty} n^{-2 / p} E^{\mathcal{F}}\left[X_{1}^{2} I\left(\left|X_{1}\right| \leq n^{1 / p}\right)\right] \\
& =\sum_{n=1}^{\infty} n^{-2 / p} \sum_{k=1}^{n} E^{\mathcal{F}}\left[X_{1}^{2} I\left((k-1)^{1 / p}<\left|X_{1}\right| \leq k^{1 / p}\right)\right] \\
& =\sum_{k=1}^{\infty} \sum_{n=k}^{\infty} n^{-2 / p} E^{\mathcal{F}}\left[X_{1}^{2} I\left((k-1)^{1 / p}<\left|X_{1}\right| \leq k^{1 / p}\right)\right] \\
& =\sum_{n=1}^{\infty} n^{-2 / p} E^{\mathcal{F}}\left[X_{1}^{2} I\left(\left|X_{1}\right| \leq 1\right)\right] \\
& +\sum_{k=2}^{\infty}\left(\sum_{n=k}^{\infty} n^{-2 / p}\right) E^{\mathcal{F}}\left[X_{1}^{2} I\left((k-1)^{1 / p}<\left|X_{1}\right| \leq k^{1 / p}\right)\right] \\
& \leq \sum_{n=1}^{\infty} n^{-2 / p}+\frac{2^{2 / p-1}}{2 / p-1} \sum_{k=2}^{\infty} k^{1-2 / p} E^{\mathcal{F}}\left[X_{1}^{2} I\left((k-1)^{1 / p}<\left|X_{1}\right| \leq k^{1 / p}\right)\right] \\
& \leq \sum_{n=1}^{\infty} n^{-2 / p}+\frac{2^{2 / p-1} p}{2-p} \\
& \times \sum_{k=2}^{\infty} k^{1-2 / p}\left(k^{1 / p}\right)^{2-p} E^{\mathcal{F}}\left[\left|X_{1}\right|^{p} I\left((k-1)^{1 / p}<\left|X_{1}\right| \leq k^{1 / p}\right)\right] \\
& =\sum_{n=1}^{\infty} n^{-2 / p}+\frac{2^{2 / p-1} p}{2-p} \sum_{k=2}^{\infty} E^{\mathcal{F}}\left[\left|X_{1}\right|^{p} I\left((k-1)^{1 / p}<\left|X_{1}\right| \leq k^{1 / p}\right)\right] \\
& \leq \sum_{n=1}^{\infty} n^{-2 / p}+\frac{2^{2 / p-1} p}{2-p} E^{\mathcal{F}}\left|X_{1}\right|^{p}<\infty \text { a.s. }
\end{aligned}
$$


Lemma 2.4. Let $p>0$. If $\left\{X_{n}, n \geq 1\right\}$ is a sequence of $\mathcal{F}$-independent and $\mathcal{F}$-identically distributed random variables, then the following statements are equivalent:

(i) $E^{\mathcal{F}}\left|X_{1}\right|^{p}<\infty$ a.s.;

(ii) $\sum_{n=1}^{\infty} P^{\mathcal{F}}\left(\left|X_{n}\right|>n^{1 / p} \varepsilon\right)<\infty$ a.s. for all $\varepsilon>0$;

(iii) $P\left(\left|X_{n}\right|>n^{1 / p} \varepsilon\right.$ i.o. $)=0$ for all $\varepsilon>0$;

(iv) $n^{-1 / p} X_{n} \rightarrow 0$ a.s.

Proof. Since the $X_{n}$ 's have the same conditional distribution, the equivalence between (i) and (ii) is evident from the estimates

$$
\begin{aligned}
E^{\mathcal{F}}\left|X_{1}\right|^{p} & =\sum_{n=1}^{\infty} E^{\mathcal{F}}\left|X_{1}\right|^{p} I\left(n-1<\left|X_{1}\right|^{p} \leq n\right) \\
& \leq \sum_{n=1}^{\infty} n P^{\mathcal{F}}\left(n-1<\left|X_{1}\right|^{p} \leq n\right) \\
& =\sum_{n=1}^{\infty} \sum_{k=1}^{n} P^{\mathcal{F}}\left(n-1<\left|X_{1}\right|^{p} \leq n\right) \\
& =\sum_{k=1}^{\infty} \sum_{n=k}^{\infty} P^{\mathcal{F}}\left(n-1<\left|X_{1}\right|^{p} \leq n\right) \\
& =\sum_{k=1}^{\infty} P^{\mathcal{F}}\left(\left|X_{1}\right|^{p}>k-1\right) \\
& \leq 1+\sum_{k=1}^{\infty} P^{\mathcal{F}}\left(\left|X_{1}\right|^{p}>k\right)
\end{aligned}
$$

and

$$
\begin{aligned}
E^{\mathcal{F}}\left|X_{1}\right|^{p} & \geq \sum_{n=1}^{\infty}(n-1) P^{\mathcal{F}}\left(n-1<\left|X_{1}\right|^{p} \leq n\right) \\
& =\sum_{n=1}^{\infty} n P^{\mathcal{F}}\left(n<\left|X_{1}\right|^{p} \leq n+1\right) \\
& =\sum_{n=1}^{\infty} \sum_{k=1}^{n} P^{\mathcal{F}}\left(n<\left|X_{1}\right|^{p} \leq n+1\right) \\
& =\sum_{k=1}^{\infty} \sum_{n=k}^{\infty} P^{\mathcal{F}}\left(n<\left|X_{1}\right|^{p} \leq n+1\right) \\
& =\sum_{k=1}^{\infty} P^{\mathcal{F}}\left(\left|X_{1}\right|^{p}>k\right) .
\end{aligned}
$$

According to the first conditional Borel-Cantelli lemma, Lemma 3.2 in Majerek et al. [7], part (ii) implies part (iii). Conversely, noting $\mathcal{F}$-independence, 
the second conditional Borel-Cantelli lemma, Lemma 3.3 in the above reference, tells us that

$$
P\left(\left|X_{n}\right|>n^{1 / p} \varepsilon \text { i.o. }\right)=P\left(\sum_{n=1}^{\infty} P^{\mathcal{F}}\left(\left|X_{n}\right|>n^{1 / p} \varepsilon\right)=\infty\right),
$$

which together with (iii) yields

$$
P\left(\sum_{n=1}^{\infty} P^{\mathcal{F}}\left(\left|X_{n}\right|>n^{1 / p} \varepsilon\right)=\infty\right)=0,
$$

so that (ii) holds.

Finally, the equivalence between (iii) and (iv) is an immediate consequence of the definition of almost sure convergence.

Proof of Theorem 2.2. Let $Y_{n}$ be defined as in (2.2). Then Lemma 2.3 assures us that

$$
\sum_{n=1}^{\infty} \operatorname{Var}^{\mathcal{F}}\left(n^{-1 / p} Y_{n}\right)<\infty \text { a.s. }
$$

which, in view of Theorem 2.1, yields

$$
\sum_{n=1}^{\infty} n^{-1 / p}\left(Y_{n}-E^{\mathcal{F}} Y_{n}\right) \text { converges a.s., }
$$

so that by the Kronecker lemma

$$
n^{-1 / p} \sum_{k=1}^{n}\left(Y_{k}-E^{\mathcal{F}} Y_{k}\right) \rightarrow 0 \text { a.s. }
$$

Next we wish to show that

$$
n^{-1 / p} \sum_{k=1}^{n} E^{\mathcal{F}} Y_{k} \rightarrow 0 \text { a.s. }
$$

in order to conclude that

$$
n^{-1 / p} \sum_{k=1}^{n} Y_{k} \rightarrow 0 \text { a.s. }
$$

First, let $0<p<1$. Some small computations yield

$$
\begin{aligned}
& \left|\sum_{k=1}^{n} E^{\mathcal{F}} Y_{k}\right| \\
\leq & \sum_{k=1}^{n} E^{\mathcal{F}}\left[\left|X_{k}\right| I\left(\left|X_{k}\right| \leq k^{1 / p}\right)\right] \\
= & \sum_{k=1}^{n} E^{\mathcal{F}}\left[\left|X_{k}\right| I\left(\left|X_{k}\right| \leq k^{1 /(2 p)}\right)\right]+\sum_{k=1}^{n} E^{\mathcal{F}}\left[\left|X_{k}\right| I\left(k^{1 /(2 p)}<\left|X_{k}\right| \leq k^{1 / p}\right)\right]
\end{aligned}
$$




$$
\begin{aligned}
\leq & \sum_{k=1}^{n}\left(k^{1 /(2 p)}\right)^{1-p} E^{\mathcal{F}}\left[\left|X_{k}\right|^{p} I\left(\left|X_{k}\right| \leq k^{1 /(2 p)}\right)\right] \\
& +\sum_{k=1}^{n}\left(k^{1 / p}\right)^{1-p} E^{\mathcal{F}}\left[\left|X_{k}\right|^{p} I\left(k^{1 /(2 p)}<\left|X_{k}\right| \leq k^{1 / p}\right)\right] \\
\leq & \sum_{k=1}^{n} k^{(1-p) /(2 p)} E^{\mathcal{F}}\left|X_{1}\right|^{p}+\sum_{k=1}^{n} k^{1 / p-1} E^{\mathcal{F}}\left[\left|X_{1}\right|^{p} I\left(\left|X_{1}\right|>k^{1 /(2 p)}\right)\right] \\
\leq & n^{(1+p) /(2 p)} E^{\mathcal{F}}\left|X_{1}\right|^{p}+\sum_{k=1}^{n} k^{1 / p-1} E^{\mathcal{F}}\left[\left|X_{1}\right|^{p} I\left(\left|X_{1}\right|>k^{1 /(2 p)}\right)\right] .
\end{aligned}
$$

Thus

$$
\begin{aligned}
n^{-1 / p}\left|\sum_{k=1}^{n} E^{\mathcal{F}} Y_{k}\right| \leq & n^{(p-1) /(2 p)} E^{\mathcal{F}}\left|X_{1}\right|^{p} \\
& +n^{-1 / p} \sum_{k=1}^{n} k^{1 / p-1} E^{\mathcal{F}}\left[\left|X_{1}\right|^{p} I\left(\left|X_{1}\right|>k^{1 /(2 p)}\right)\right],
\end{aligned}
$$

which converges to 0 almost surely, since the first term in the right-hand side converging to 0 almost surely is evident, and the second term converging to 0 almost surely from Lemma A.6.1 in Gut [4] and the observations

$$
\begin{aligned}
& n^{-1 / p} \sum_{k=1}^{n} k^{1 / p-1} E^{\mathcal{F}}\left[\left|X_{1}\right|^{p} I\left(\left|X_{1}\right|>k^{1 /(2 p)}\right)\right] \\
\leq & \frac{1}{\sum_{k=1}^{n} k^{1 / p-1}} \sum_{k=1}^{n} k^{1 / p-1} E^{\mathcal{F}}\left[\left|X_{1}\right|^{p} I\left(\left|X_{1}\right|>k^{1 /(2 p)}\right)\right]
\end{aligned}
$$

and

$$
E^{\mathcal{F}}\left[\left|X_{1}\right|^{p} I\left(\left|X_{1}\right|>k^{1 /(2 p)}\right)\right] \rightarrow 0 \text { a.s. as } k \rightarrow \infty .
$$

Next, let $1 \leq p<2$. Then, by the assumption of $E^{\mathcal{F}} X_{1}=0$ a.s.,

$$
\begin{aligned}
\left|\sum_{k=1}^{n} E^{\mathcal{F}} Y_{k}\right| & =\left|\sum_{k=1}^{n} E^{\mathcal{F}}\left[X_{k} I\left(\left|X_{k}\right|>k^{1 / p}\right)\right]\right| \\
& \leq \sum_{k=1}^{n} E^{\mathcal{F}}\left[\left|X_{k}\right| I\left(\left|X_{k}\right|>k^{1 / p}\right)\right] \\
& \leq \sum_{k=1}^{n}\left(k^{1 / p}\right)^{1-p} E^{\mathcal{F}}\left[\left|X_{1}\right|^{p} I\left(\left|X_{1}\right|>k^{1 / p}\right)\right] \\
& =\sum_{k=1}^{n} k^{1 / p-1} E^{\mathcal{F}}\left[\left|X_{1}\right|^{p} I\left(\left|X_{1}\right|>k^{1 / p}\right)\right],
\end{aligned}
$$


which means

$$
n^{-1 / p}\left|\sum_{k=1}^{n} E^{\mathcal{F}} Y_{k}\right| \leq n^{-1 / p} \sum_{k=1}^{n} k^{1 / p-1} E^{\mathcal{F}}\left[\left|X_{1}\right|^{p} I\left(\left|X_{1}\right|>k^{1 / p}\right)\right] .
$$

Since $1 / 2<1 / p \leq 1, \sum_{k=1}^{n} k^{1 / p-1} \leq p n^{1 / p}$ by Lemma A.3.1 in Gut [4]. Again by using Lemma A.6.1 in Gut [4], we can continue (2.5) to get

$$
\begin{aligned}
n^{-1 / p}\left|\sum_{k=1}^{n} E^{\mathcal{F}} Y_{k}\right| & \leq \frac{p}{\sum_{k=1}^{n} k^{1 / p-1}} n^{-1 / p} \sum_{k=1}^{n} k^{1 / p-1} E^{\mathcal{F}}\left[\left|X_{1}\right|^{p} I\left(\left|X_{1}\right|>k^{1 / p}\right)\right] \\
& \rightarrow 0 .
\end{aligned}
$$

In both cases, (2.3) and hence (2.4) holds.

Finally, from $E^{\mathcal{F}}\left|X_{1}\right|^{p}<\infty$ a.s. and Lemma 2.4 we have

$$
P\left(\left|X_{n}\right|>n^{1 / p} \text { i.o. }\right)=0,
$$

or, equivalently,

$$
P\left(X_{n} \neq Y_{n} \text { i.o. }\right)=0
$$

which indicates that $\sum_{n=1}^{\infty}\left(X_{n}-Y_{n}\right)$ converges almost surely, and hence we complete the proof of $(2.1)$.

Remark 2.5. If $0<p<1$ and $\left\{X_{n}, n \geq 1\right\}$ is a sequence of identically distributed random variables with $E\left|X_{1}\right|^{p}<\infty$, then $\sum_{n=1}^{\infty} n^{-1 / p}\left|X_{n}\right|<\infty$ a.s. (see Lemma 3.2.1 of Chandra [1]). Hence (2.1) holds irrespective of any dependence condition. In Theorem 2.2, although the assumption of $\mathcal{F}$-identical distribution is stronger than identical distribution of $\left\{X_{n}, n \geq 1\right\}$, the assumption of $E^{\mathcal{F}}\left|X_{1}\right|^{p}<\infty$ a.s. is weaker than $E\left|X_{1}\right|^{p}<\infty$.

Now we consider the converse of the conditional Marcinkiewicz-Zygmund strong law.

Theorem 2.6. Suppose that $\left\{X_{n}, n \geq 1\right\}$ is a sequence of $\mathcal{F}$-independent and $\mathcal{F}$-identically distributed random variables. If for some $\mathcal{F}$-measurable random variable $Y$ and for some $p \in(0,2)$,

$$
n^{-1 / p}\left(S_{n}-n Y\right) \rightarrow 0 \text { a.s., }
$$

then $E^{\mathcal{F}}\left|X_{1}\right|^{p}<\infty$ a.s. and $E^{\mathcal{F}} X_{1}=Y$ a.s. when $1 \leq p<2$.

Proof. Note that

$$
\begin{aligned}
n^{-1 / p} X_{n}= & n^{-1 / p} Y+n^{-1 / p}\left(S_{n}-n Y\right) \\
& -[(n-1) / n]^{1 / p}(n-1)^{-1 / p}\left[S_{n-1}-(n-1) Y\right] \rightarrow 0 \text { a.s. }
\end{aligned}
$$

so that $E^{\mathcal{F}}\left|X_{1}\right|^{p}<\infty$ a.s. by Lemma 2.4. Moreover, if $1 \leq p<2$, then

$$
n^{-1} S_{n}=n^{1 / p-1} n^{-1 / p}\left(S_{n}-n Y\right)+Y \rightarrow Y \text { a.s. }
$$

Via an appeal to the necessity part of Theorem 4.2 in Majerek et al. [7], we thus get $E^{\mathcal{F}} X_{1}=Y$. 


\section{Conditional versions of Marcinkiewicz-Zygmund inequalities and convergence in $L^{p}$}

We first restate, in a slightly modified form, Theorem 4.1 of [12], which is a conditional version of Fubini's theorem.

Lemma 3.1. Let $X(\cdot, \cdot): \Omega \times \mathbb{R} \rightarrow \mathbb{R}$ be $\mathcal{A} \times \mathcal{B}$-measurable and either nonnegative or $P \times \mu$-integrable, where $\mu$ is the Lebesgue measure. Then

$$
E^{\mathcal{F}} \int_{R} X(\cdot, t) d t=\int_{R} E^{\mathcal{F}} X(\cdot, t) d t \text { a.s. }
$$

Lemma 3.2 is a simple consequence of Lemma 1 in Prakasa Rao [9].

Lemma 3.2. Let $p>1$ and let $X_{1}, X_{2}, \ldots, X_{n}$ be $\mathcal{F}$-independent random variables with $E^{\mathcal{F}} X_{k}=0$ a.s. for all $1 \leq k \leq n$. If $\varepsilon_{1}, \varepsilon_{2}, \ldots, \varepsilon_{n}$ are given with $\varepsilon_{k}= \pm 1$ for all $k$, then

$$
E^{\mathcal{F}}\left|\sum_{k=1}^{n} \varepsilon_{k} X_{k}\right|^{p} \leq 2^{p} E^{\mathcal{F}}\left|\sum_{k=1}^{n} X_{k}\right|^{p} \quad \text { a.s. }
$$

Proof. We may (by relabeling indices if necessary) assume that $\varepsilon_{1}=\cdots=\varepsilon_{j}=$ $1, \varepsilon_{j+1}=\cdots=\varepsilon_{n}=-1$ for some $j \in\{1, \ldots, n-1\}$ and then define

$$
X^{\prime}=\sum_{k=1}^{j} X_{k}, \quad X^{\prime \prime}=\sum_{k=j+1}^{n} X_{k} .
$$

Clearly $X^{\prime}+X^{\prime \prime}=\sum_{k=1}^{n} X_{k}$ and $X^{\prime}-X^{\prime \prime}=\sum_{k=1}^{n} \varepsilon_{k} X_{k}$. Our assumptions imply that $E^{\mathcal{F}} X^{\prime}=E^{\mathcal{F}} X^{\prime \prime}=0$ a.s. and $X^{\prime}$ and $X^{\prime \prime}$ are $\mathcal{F}$-independent, whence $E^{\mathcal{F}}\left|X^{\prime}\right|^{p} \leq E^{\mathcal{F}}\left|X^{\prime}+X^{\prime \prime}\right|^{p}$ and $E^{\mathcal{F}}\left|X^{\prime \prime}\right|^{p} \leq E^{\mathcal{F}}\left|X^{\prime}+X^{\prime \prime}\right|^{p}$ from Lemma 1 of Prakasa Rao [9]. The conditional Minkowski inequality applies and gives

$$
\begin{aligned}
\left(E^{\mathcal{F}}\left|\sum_{k=1}^{n} \varepsilon_{k} X_{k}\right|^{p}\right)^{1 / p} & =\left(E^{\mathcal{F}}\left|X^{\prime}-X^{\prime \prime}\right|^{p}\right)^{1 / p} \\
& \leq\left(E^{\mathcal{F}}\left|X^{\prime}\right|^{p}\right)^{1 / p}+\left(E^{\mathcal{F}}\left|X^{\prime \prime}\right|^{p}\right)^{1 / p} \\
& \leq 2\left(E^{\mathcal{F}}\left|X^{\prime}+X^{\prime \prime}\right|^{p}\right)^{1 / p} \\
& =2\left(E^{\mathcal{F}}\left|\sum_{k=1}^{n} X_{k}\right|^{p}\right)^{1 / p}
\end{aligned}
$$

This yields the desired result.

Next let us recall Khintchine's inequality (see e.g. Rosenthal [11]). Suppose that $r(t)$ is the function with period one defined on the real line by $r(t)=1$ or -1 according as $0 \leq t<1 / 2$ or $1 / 2 \leq t<1$ and put $r_{k}(t)=r\left(2^{k-1} t\right)$, 
$k=1,2, \ldots, n$, the first $n$ Rademacher functions. Then, for any $p>0$, there exist constants $A_{p}$ and $B_{p}$ depending only $p$, so that for any $n$ scalars $c_{1}, \ldots, c_{n}$,

$$
A_{p}\left(\sum_{k=1}^{n} c_{k}^{2}\right)^{p / 2} \leq \int_{0}^{1}\left|\sum_{k=1}^{n} c_{k} r_{k}(t)\right|^{p} d t \leq B_{p}\left(\sum_{k=1}^{n} c_{k}^{2}\right)^{p / 2} .
$$

With the help of the above lemmas, we can establish the conditional versions of Marcinkiewicz-Zygmund inequalities, its unconditional version is Theorem 3.8.1 of Gut [4].

Theorem 3.3. Let $p \geq 1$. Suppose that $X_{1}, X_{2}, \ldots, X_{n}$ are $\mathcal{F}$-independent random variables with $\bar{E}^{\mathcal{F}}\left|X_{k}\right|^{p}<\infty$ a.s. and $E^{\mathcal{F}} X_{k}=0$ a.s. for all $1 \leq k \leq$ $n$. Then there exist constants $A_{p}^{*}$ and $B_{p}^{*}$ depending only on $p$ such that

$$
A_{p}^{*} E^{\mathcal{F}}\left(\sum_{k=1}^{n} X_{k}^{2}\right)^{p / 2} \leq E^{\mathcal{F}}\left|\sum_{k=1}^{n} X_{k}\right|^{p} \leq B_{p}^{*} E^{\mathcal{F}}\left(\sum_{k=1}^{n} X_{k}^{2}\right)^{p / 2} \text { a.s. }
$$

In particular,

$$
A_{p}^{*} E\left(\sum_{k=1}^{n} X_{k}^{2}\right)^{p / 2} \leq E\left|\sum_{k=1}^{n} X_{k}\right|^{p} \leq B_{p}^{*} E\left(\sum_{k=1}^{n} X_{k}^{2}\right)^{p / 2} .
$$

Proof. Relation (3.3) follows from (3.2) by taking expectations, so we need only to prove (3.2). From Lemma 3.2,

$$
2^{-p} E^{\mathcal{F}}\left|\sum_{k=1}^{n} r_{k}(t) X_{k}\right|^{p} \leq E^{\mathcal{F}}\left|\sum_{k=1}^{n} X_{k}\right|^{p} \leq 2^{p} E^{\mathcal{F}}\left|\sum_{k=1}^{n} r_{k}(t) X_{k}\right|^{p} \text { a.s. }
$$

Integrating the left-most inequality in (3.4) with respect to $t$ from 0 to 1 and using Lemma 3.1, we obtain by (3.1) that

$$
\begin{aligned}
E^{\mathcal{F}}\left|\sum_{k=1}^{n} X_{k}\right|^{p} & \geq 2^{-p} \int_{0}^{1} E^{\mathcal{F}}\left|\sum_{k=1}^{n} r_{k}(t) X_{k}\right|^{p} d t \\
& =2^{-p} E^{\mathcal{F}} \int_{0}^{1}\left|\sum_{k=1}^{n} r_{k}(t) X_{k}\right|^{p} d t \geq 2^{-p} A_{p} E^{\mathcal{F}}\left(\sum_{k=1}^{n} X_{k}^{2}\right)^{p / 2} \text { a.s. }
\end{aligned}
$$

In the same way, the right-most inequality in (3.4) yields

$$
\begin{aligned}
E^{\mathcal{F}}\left|\sum_{k=1}^{n} X_{k}\right|^{p} & \leq 2^{p} \int_{0}^{1} E^{\mathcal{F}}\left|\sum_{k=1}^{n} r_{k}(t) X_{k}\right|^{p} d t \\
& =2^{p} E^{\mathcal{F}} \int_{0}^{1}\left|\sum_{k=1}^{n} r_{k}(t) X_{k}\right|^{p} d t \leq 2^{p} B_{p} E^{\mathcal{F}}\left(\sum_{k=1}^{n} X_{k}^{2}\right)^{p / 2} \text { a.s. }
\end{aligned}
$$

Hence (3.2) follows from the last two inequalities with $A_{p}^{*}=2^{-p} A_{p}$ and $B_{p}^{*}=$ $2^{p} B_{p}$, where $A_{p}$ and $B_{p}$ are the constants in Khintchine's inequality. 
As an application of Theorem 3.3, we can derive a result on convergence in $L^{p}$, which is worth comparing with Theorem 2.2.

Theorem 3.4. Let $0<p<2$. Suppose that $\left\{X_{n}, n \geq 1\right\}$ is a sequence of $\mathcal{F}$-independent and $\mathcal{F}$-identically distributed random variables. Then

$$
E\left|X_{1}\right|^{p}<\infty \text {, and } E^{\mathcal{F}} X_{1}=0 \text { a.s. when } 1 \leq p<2
$$

if and only if

$$
n^{-1 / p} S_{n} \rightarrow 0 \text { in } L^{p}
$$

Proof. We first prove the necessity. Let $\varepsilon$ be a positive number, fixed for a moment. Let us choose a positive number $M$ that is enough large to satisfy $E\left|X_{1}\right|^{p} I\left(\left|X_{1}\right|>M\right)<\varepsilon$, and then set

$$
Y_{k}=X_{k} I\left(\left|X_{k}\right| \leq M\right), Z_{k}=X_{k} I\left(\left|X_{k}\right|>M\right), \quad k \geq 1 .
$$

Thus, in the case where $0<p<1$, one obtains

$E\left|S_{n}\right|^{p} \leq E\left|\sum_{k=1}^{n} Y_{k}\right|^{p}+E\left|\sum_{k=1}^{n} Z_{k}\right|^{p} \leq E\left|\sum_{k=1}^{n} Y_{k}\right|^{p}+\sum_{k=1}^{n} E\left|Z_{k}\right|^{p} \leq(n M)^{p}+n \varepsilon$,

so that $\limsup _{n \rightarrow \infty} E\left(n^{-1}\left|S_{n}\right|^{p}\right) \leq \varepsilon$. This is true for any $\varepsilon>0$, so it follows that $\lim _{n \rightarrow \infty} E\left(n^{-1}\left|S_{n}\right|^{p}\right)=0$, namely $n^{-1 / p} S_{n} \rightarrow 0$ in $L^{p}$.

If $1 \leq p<2$, then we get by Theorem 3.3 that

$$
\begin{aligned}
E\left|S_{n}\right|^{p} & \leq B_{p}^{*} E\left(\sum_{k=1}^{n} X_{k}^{2}\right)^{p / 2} \\
& \leq 2^{p / 2} B_{p}^{*} E\left(\sum_{k=1}^{n}\left(Y_{k}^{2}+Z_{k}^{2}\right)\right)^{p / 2} \\
& \leq 2^{p / 2} B_{p}^{*}\left[E\left(\sum_{k=1}^{n} Y_{k}^{2}\right)^{p / 2}+E\left(\sum_{k=1}^{n} Z_{k}^{2}\right)^{p / 2}\right] \\
& \leq 2^{p / 2} B_{p}^{*}\left[\left(n M^{2}\right)^{p / 2}+n E\left|Z_{1}\right|^{p}\right] \\
& \leq 2^{p / 2} B_{p}^{*}\left(n^{p / 2} M^{p}+n \varepsilon\right)
\end{aligned}
$$

after which the desired conclusion follows as for the case $0<p<1$.

Next we prove the sufficiency. Define $C_{p}=1$ if $0<p<1$ and $2^{p-1}$ if $1 \leq p<2$, then the assumption $n^{-1} E\left|S_{n}\right|^{p} \rightarrow 0$ yields

$\frac{1}{n} E\left|X_{1}\right|^{p} \leq \frac{1}{n} E\left|S_{n}-S_{n-1}\right|^{p} \leq C_{p}\left[\frac{1}{n} E\left|S_{n}\right|^{p}+\frac{n-1}{n} \cdot \frac{1}{n-1} E\left|S_{n-1}\right|^{p}\right] \rightarrow 0$, which forces that $E\left|X_{1}\right|^{p}<\infty$.

For any $\varepsilon>0$, we have

$$
P\left(n^{-1 / p}\left|S_{n}\right|>\varepsilon\right) \leq \varepsilon^{-p} n^{-1} E\left|S_{n}\right|^{p} \rightarrow 0,
$$


which means $n^{-1 / p} S_{n} \rightarrow 0$ in probability and hence $n^{-1} S_{n} \rightarrow 0$ in probability when $1 \leq p<2$. But Theorem 4.2 tells us that $n^{-1} S_{n} \rightarrow E^{\mathcal{F}} X_{1}$ a.s., so that $E^{\mathcal{F}} X_{1}=0$ a.s.

There exists a variant of Theorem 3.4, we still give its proof for the sake of completeness.

Theorem 3.5. Let $0<p<2$. Suppose that $\left\{X_{n}, n \geq 1\right\}$ is a sequence of $\mathcal{F}$-independent and $\mathcal{F}$-identically distributed random variables. Then

$$
E^{\mathcal{F}}\left|X_{1}\right|^{p}<\infty \text {, and } E^{\mathcal{F}} X_{1}=0 \text { a.s. when } 1 \leq p<2
$$

if and only if

$$
n^{-1} E^{\mathcal{F}}\left|S_{n}\right|^{p} \rightarrow 0 \text { a.s. }
$$

Proof. We first prove the necessity. Let $M$ be a positive number, fixed for a moment, and then define $Y_{k}, Z_{k}$ as in (3.5).

In case $0<p<1$, one has

$E^{\mathcal{F}}\left|S_{n}\right|^{p} \leq E^{\mathcal{F}}\left|\sum_{k=1}^{n} Y_{k}\right|^{p}+\sum_{k=1}^{n} E^{\mathcal{F}}\left|Z_{k}\right|^{p} \leq(n M)^{p}+n E^{\mathcal{F}}\left[\left|X_{1}\right|^{p} I\left(\left|X_{1}\right|>M\right)\right]$, so that $\limsup _{n \rightarrow \infty} n^{-1} E^{\mathcal{F}}\left|S_{n}\right|^{p} \leq E^{\mathcal{F}}\left[\left|X_{1}\right|^{p} I\left(\left|X_{1}\right|>M\right)\right]$. Letting $M$ approach infinity, we get the desired conclusion.

In case $1 \leq p<2$, Theorem 3.3 applies and gives

$$
\begin{aligned}
E^{\mathcal{F}}\left|S_{n}\right|^{p} & \leq B_{p}^{*} E^{\mathcal{F}}\left(\sum_{k=1}^{n} X_{k}^{2}\right)^{p / 2} \\
& \leq 2^{p / 2} B_{p}^{*}\left\{n^{p / 2} M^{p}+n E^{\mathcal{F}}\left[\left|X_{1}\right|^{p} I\left(\left|X_{1}\right|>M\right)\right]\right\} .
\end{aligned}
$$

Proceeding as in the last part of the proof in case $0<p<1$, the desired conclusion follows as $1 / 2<p / 2<1$.

Next we prove the sufficiency. Define $C_{p}$ as in the proof of Theorem 3.4, then the assumption $n^{-1} E^{\mathcal{F}}\left|S_{n}\right|^{p} \rightarrow 0$ a.s. yields

$$
\begin{aligned}
\frac{1}{n} E^{\mathcal{F}}\left|X_{1}\right|^{p} & \leq \frac{1}{n} E^{\mathcal{F}}\left|S_{n}-S_{n-1}\right|^{p} \\
& \leq C_{p}\left[\frac{1}{n} E^{\mathcal{F}}\left|S_{n}\right|^{p}+\frac{n-1}{n} \cdot \frac{1}{n-1} E^{\mathcal{F}}\left|S_{n-1}\right|^{p}\right] \rightarrow 0 \text { a.s. }
\end{aligned}
$$

which forces that $E^{\mathcal{F}}\left|X_{1}\right|^{p}<\infty$ a.s.

For any $\varepsilon>0$, it is easy to see

$$
P^{\mathcal{F}}\left(n^{-1 / p}\left|S_{n}\right|>\varepsilon\right) \leq \varepsilon^{-p} n^{-1} E^{\mathcal{F}}\left|S_{n}\right|^{p} \rightarrow 0 \text { a.s. },
$$

which and the dominated convergence theorem yield $P\left(n^{-1 / p}\left|S_{n}\right|>\varepsilon\right) \rightarrow 0$, namely $n^{-1 / p} S_{n} \rightarrow 0$ in probability. This means $n^{-1} S_{n} \rightarrow 0$ in probability when $1 \leq p<2$, after which the conclusion $E^{\mathcal{F}} X_{1}=0$ a.s. follows as in the last part of the proof of Theorem 3.4. 
Theorem 3.5 together with Theorems 2.2 and 2.6 yields the following implications, which are the conditional versions of the main results in Pyke and Root [10] under the non-conditional case.

Theorem 3.6. Suppose that $\left\{X_{n}, n \geq 1\right\}$ is a sequence of $\mathcal{F}$-independent and $\mathcal{F}$-identically distributed random variables. For each $p \in(0,2)$, the following statements are equivalent:

(i) $E^{\mathcal{F}}\left|X_{1}\right|^{p}<\infty$ a.s. (assume $E^{\mathcal{F}} X_{1}=0$ a.s. if $1 \leq p<2$ );

(ii) $n^{-1 / p} S_{n} \rightarrow 0$ a.s.;

(iii) $n^{-1} E^{\mathcal{F}}\left|S_{n}\right|^{p} \rightarrow 0$ a.s.

Remark 3.7. Theorem 3.6 remains valid provided that $\left\{X_{n}, n \geq 1\right\}$ is a sequence of exchangeable random variables and $\mathcal{F}$ is taken as the tail $\sigma$-algebra or the $\sigma$-algebra of permutable events of such a sequence. To the best of our knowledge, this result has not been established previously in the literature.

\section{References}

[1] T. K. Chandra, Laws of Large Numbers, New Delhi, Narosa Publishing House, 2012.

[2] Y. S. Chow and H. Teicher, Probability Theory: Independence, Interchangeability, Martingales, 3rd Edition, New York, Springer-Verlag, 1997.

[3] T. C. Christofides and M. Hadjikyriakou, Conditional demimartingales and related results, J. Math. Analy. Appl. 398 (2013), no. 1, 380-391.

[4] A. Gut, Probability: A graduate course, 2nd Edition, New York, Springer-Verlag, 2013.

[5] J. C. Liu and B. L. S. Prakasa Rao, On conditional Borel-Cantelli lemmas for sequences of random variables, J. Math. Anal. Appl. 399 (2013), no. 1, 156-165.

[6] J. C. Liu and L. D. Zhang, Conditional Borel-Cantelli lemma and conditional strong law of large number, Acta Math. Appl. Sin. (Chinese Ser.) 37 (2014), no. 3, 537-546.

[7] D. Majerek, W. Nowak, and W. Zieba, Conditional strong law of large number, Int. J. Pure Appl. Math. 20 (2005), no. 2, 143-157.

[8] M. Ordóñez Cabrera, A. Rosalsky, and A. Volodin, Some theorems on conditional mean convergence and conditional almost sure convergence for randomly weighted sums of dependent random variables, TEST 21 (2012), no. 2, 369-385.

[9] B. L. S. Prakasa Rao, Conditional independence, conditional mixing and conditional association, Ann. Inst. Statist. Math. 61 (2009), no. 2, 441-460.

[10] R. Pyke and D. Root, On convergence in $r$-mean for normalized partial sums, Ann. Math. Statist. 39 (1968), no. 2, 379-381.

[11] H. P. Rosenthal, On the subspaces of $L^{p}(p>2)$ spanned by sequences of independent random variables, Israel J. Math. 8 (1970), no. 3, 273-303.

[12] G. G. Roussas, On conditional independence, mixing, and association, Stoch. Anal. Appl. 26 (2008), no. 6, 1274-1309.

[13] A. N. Shiryaev, Probability, 2nd Edition, New York, Springer-Verlag, 1996.

[14] X. H. Wang and X. J. Wang, Some inequalities for conditional demimartingales and conditional N-demimartingales, Statist. Probab. Lett. 83 (2013), no. 3, 700-709.

[15] D. M. Yuan, J. An, and X. S. Wu, Conditional limit theorems for conditionally negatively associated random variables, Monatsh. Math. 161 (2010), no. 4, 449-473.

[16] D. M. Yuan, X. M. Hu, and B. Tao, Some results on conditionally uniformly strong mixing sequences of random variables, J. Korean Math. Soc. 51 (2014), no. 3, 609-633.

[17] D. M. Yuan and L. Lei, Some conditional results for conditionally strong mixing sequences of random variables, Sci. China Math. 56 (2013), no. 4, 845-859. 
[18] D. M. Yuan, L. R. Wei, and L. Lei, Conditional central limit theorems for a sequence of conditional independent random variables, J. Korean Math. Soc. 51 (2014), no. 1, 1-15.

[19] D. M. Yuan and Y. Xie, Conditional limit theorems for conditionally linearly negative quadrant dependent random variables, Monatsh. Math. 166 (2012), no. 2, 281-299.

[20] D. M. Yuan and Y. K. Yang, Conditional versions of limit theorems for conditionally associated random variables, J. Math. Anal. Appl. 376 (2011), no. 1, 282-293.

DE-MEI YuAN

School of Mathematics and Statistics

Chongqing Technology and Business University

Chongqing 400067, P. R. China

E-mail address: yuandemei@163.com

SHUN-JING LI

School of Mathematics and Statistics

Chongling Technology and Business University

Chongqing 400067, P. R. China

E-mail address: $895430886 @$ qq.com 